\title{
Neuartige Gold-Nanokomposite für chemiresistive Sensoren
}

\author{
Yelyena Daskal ${ }^{1}$, Rosemarie Dittrich ${ }^{1}$, Cameliu Himcinschi , Günter Gärtner ${ }^{3}$, Barbara Abendroth ${ }^{3}$, Juliane \\ Walter ${ }^{3}$, Yvonne Joseph ${ }^{1}$ \\ ${ }^{1}$ Institut für Elektronik- und Sensormaterialien, TU Bergakademie Freiberg,Freiberg, Deutschland \\ ${ }^{2}$ Institut für Theoretische Physik, TU Bergakademie Freiberg, Freiberg, Deutschland \\ ${ }^{3}$ Institut für Experimentelle Physik, TU Bergakademie Freiberg, Freiberg, Deutschland \\ Kontakt: yedaskal@gmail.com
}

\section{Zusammenfassung:}

Wir haben neuartige Goldnanopartikelnnetzwerke mit verschiedenen aromatischen Molekülen $(1,4-$ Benzenedithiol, 1,4-Benzenedimethanethiol, Biphenyl-4,4'-dithiol und p-Terphenyl-4,4"-dithiol) verlinkt auf silanisierten Glas oder Siliziumsubstraten entweder automatisch mit einer Durchflußzelle, oder manuell durch aufschleudern und lagenweise Selbstassemblierung hergestellt. Die resultierenden dünnen Filme sind mittels Röntgen-Photoelektronenspektroskopie charakterisiert worden. Die Messungen zeigten die chemische Zusammensetzung sowie den Vernetzungsgrad der Komposite. Oberflächenverstärkte Ramanspektroskopie, oberflächenverstärkte Infrarot-Absorption, Ellipsometrie und die UV/VisSpektroskopie wurden verwendet, um die optischen Eigenschaften der Komposite zu vermessen. Schließlich wurden die chemisensorischen Eigenschaften der Schichten mit verschiedenen volatilen organischen Komponenten (VOC) (Toluol, Wasser, 1-Propanol, 4-Methyl-2-Pentanon) in Konzentrationen von 100 bis $5000 \mathrm{ppm}$ charakterisiert. Die resultierenden Sensorantworten sind für alle Analyten jedoch deutlich kleiner als für Sensoren mit aliphatischen organischen Molekülen, was auf einen veränderten Sensormechanismus oder Ladungstransport zwischen den Partikeln schließen lässt.

Schlagwörter: Gold, Nanopartikeln, Sensor, Röntgen-Photoelektronenspektroskopie (XPS) Oberflächenverstärkte Ramanspektroskopie (SERS), oberflächenverstärkte Infrarot-Absorption (SEIRA).

\section{Einleitung}

Komposite aus metallischen Nanopartikeln und organischen Molekülen haben in den letzten Jahren in der Materialforschung erhebliche Aufmerksamkeit erfahren. Insbesondere im Bereich von chemischen Sensoren sind Nanopartikelnetzwerke für den Nachweis von Gasen und Dämpfen sehr gut geeignet. Zahlreiche Studien demonstrierten bereits Herstellungs- und Messverfahren für Materialien basierend auf GoldNanopartikeln, die mit aliphatischen Dithiolen vernetzt sind. [1-3]

In dieser Arbeit berichten wir über die Assemblierung, Materialleitfähigkeit, chemische Zusammensetzung und die Dampfreaktion der Nanopartikel-Netzwerke, die mit den aromatischen, leitfähigen organischen Molekülen miteinander verknüpft sind. Als Nanopartikelsbestandteil wurden Dodecylaminstabilisierte Goldnanopartikeln verwendet [4]. Aufgrund von Austauschreaktionen mit den organischen Linkermolekülen (1,4-Benzenedithiol, 1,4-Benzenedimethanethiol, Biphenyl-4,4'-dithiol und p-Terphenyl-4,4"-dithiol) werden Goldnanopartikeln Layer-by-Layer verlinkt.

\section{Methoden und Materialien}

Chemikalien wurden von Sigma Aldrich, Merck, VWR, Technic France und Honeywell bezogen. Alle Chemikalien waren p. A.-Qualität. 1,4-
Benzenedithiol, 1,4-Benzenedimethanethiol, Biphenyl-4,4'-dithiol und p-Terphenyl-4,4"-dithiol wurden als die organischen Linkermoleküle $(0,5$ $\mathrm{mmol} / \mathrm{L}$ mit Toluol) verwendet. 3Mercaptopropyltriethoxysilan (MPTES) wurde als Silanisierungsmittel für die Funktionalisierung der Substratoberfläche angewendet. Die Dodecylamingeschützten Goldnanopartikeln (AuNP) wurden bei $20^{\circ} \mathrm{C}$ [4] synthetisiert. Entionisiertes (DI)-Wasser mit einem Widerstand von $1 \mathrm{M} \Omega . \mathrm{cm}$ (Purite HP700 lonenaustauscher) wurde während der Experimente verwendet. Ein Siliziumwafer mit definierten Dicke von $625 \pm 15 \mu \mathrm{m}$ wurde von Siltronic AG bezogen und geschnitten. Die Siliciumstreifen mit der Kantenlänge ca. $10 \mathrm{~mm}$ und $15 \mathrm{~mm}$ wurden als Substrate für die Messungen genutzt.

Die Substrate für Sensormessungen wurden mit goldenen Fingerelektroden (52 Fingerpaare, 100 $\mu \mathrm{m}$ Breite und 5,2 $\mathrm{mm}$ Länge, $50 \mu \mathrm{m}$ Abstand) ausgestattet.

\section{Manuelle Herstellung durch Aufschleudern:}

Die manuelle Herstellung der Kompositschichten erfolgte durch Selbstassemblierung, indem lagenweise Goldnanopartikeln und organische Linker mittels Spin coater aufgeschleudert wurden.

Die Probenvorbereitung wurde von Schlicke u.a. vorgeschlagen [5] und in einer modifizierten Weise verwendet, wie in [6] berichtet. Die fertigen Proben wurden unter Umgebungsbedingungen gelagert. 
Automatische Herstellung mit einer Durchflußzelle:

Die Methode wurde zuvor von D. Bethell und Mitarbeiter [7] beschrieben. Zunächst wurden die Siliciumsubstrate mit DI-Wasser und Isopropanol gereinigt und in der Linkerlösung über Nacht belassen. Vor dem Filmauftragen wurden die Substratflächen durch Gasphasensilanisierung funktionalisiert. Dann wurden die Substrate in die Fluidmesszelle eingesetzt, 5 Minuten mit Nanopartikelnlösung behandelt, dann 4 Minuten lang mit Toluol gespült, 5 Minuten mit dem Linkerlösung nachbehandelt und noch einmal mit Toluol gespült (1 Abscheidungszyklus). Diese Schritte wurden 15-mal wiederholt. Danach wurden die Filme mit Toluol abgespült und 10 Minuten mit Luft getrocknet. Nach der Herstellung wurden alle Proben in einen Exsikkator unter Argonatmosphäre gegeben.

\section{Ergebnisse und Diskussion}

\section{UV/Vis-Spektroskopie}

Um die Absorptionsspektren der Linker zu beobachten, wurde ein Zeiss Specord S10Spektrometer verwendet. Für unsere Messungen wurde der Extinktion-Modus ausgewählt, die Anzahl der Akkumulationen war gleich 10 und Integrationszeit wurde zu 83,7 ms abgegeben.

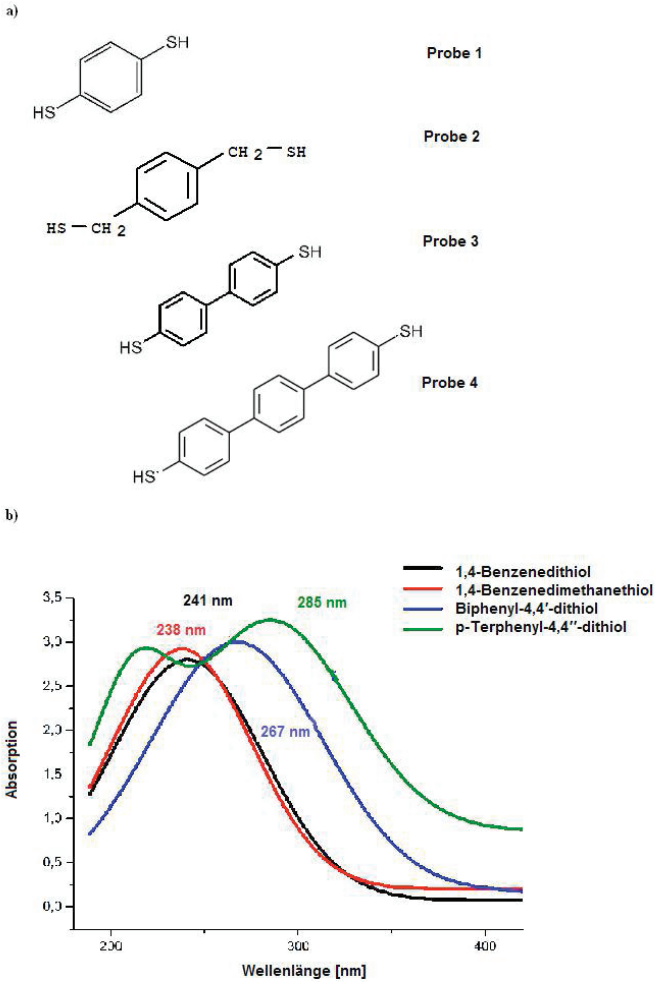

Abb. 1: a) Molekülformel der Linker; b) Uv/vis Spektren der Linker.
In allen Fällen wurden die Extinktion und die Position der Spitze der Oberflächenplasmonbande (SPB) bestimmt. Für Messungen von Flüssigkeitsproben hat die Weglänge $1 \mathrm{~cm}$ betragen.

Die Positionen des Absorptionsmaximums und Band-Cutoff der Spektren sind in Tab. 1. zusammengefasst. Beide Werte verschieben sich zu den höheren Wellenlängen mit zunehmender Anzahl der Phenylringe.

Tab.1: UV-VIS-Bänder, Band-Cutoffs und Energien von HOMO / LUMO-Übergängen der angegebenen Moleküle (1), ausgerechnet von den UV-VIS-Band Cutoffs (2) von der Ref. [8] und (3) Ref. [9].

\begin{tabular}{|c|r|r|r|r|}
\hline & $\begin{array}{c}\text { Pro } \\
\text { be 1 }\end{array}$ & $\begin{array}{c}\text { Pro } \\
\text { be 2 }\end{array}$ & $\begin{array}{c}\text { Pro } \\
\text { be 3 }\end{array}$ & $\begin{array}{c}\text { Pro } \\
\text { be 4 }\end{array}$ \\
\hline$\lambda_{\text {max }}$ [nm] & 238 & 241 & 267 & 285 \\
\hline$\lambda_{\text {cutoff }}$ [nm] & 326 & 336 & 375 & 393 \\
\hline $\begin{array}{l}\text { HOMO- } \\
\text { LUMO Lücke } \\
\text { Exp }_{\text {exp) }} \text { (eV }\end{array}$ & 3,67 & 3,56 & 3,19 & 3,04 \\
\hline $\begin{array}{l}\text { HOMO- } \\
\text { LUMO Lücke } \\
\text { Ekalk }_{\text {(eV) }}{ }^{2}\end{array}$ & 2,66 & - & 2,57 & 2,44 \\
\hline $\begin{array}{l}\text { HOMO- } \\
\text { LUMO Lücke } \\
\text { Ek }\end{array}$ & - & 4,0 & & \\
\hline
\end{tabular}

\section{Ellipsometrie:}

Ellipsometriemessungen wurden unter Verwendung eines M2000 KompensatorEllipsometers aufgenommen. (J. A. Woollam Co, Lincoln, NE). Die Messungen wurden unter Einfallswinkeln $70^{\circ}$ und $75^{\circ}$ in Bezug auf die Substratnormale durchgeführt. Die Spektren der Brechzahl $n(\lambda)$ und des Extinktionskoeffizienten $k$ ( $\lambda$ ) (Abb. 2) der Dünnfilme wurden aus den ellipsometrischen Daten erhalten. Wir schlagen vor, dass das Modell unsere Proben als getrennte Goldnanopartikeln in der organischen Matrix beschreibt. Um die dielektrische Funktion der Goldnanopartikeln zu beschreiben, verwanden wir das EMA-Modell. Für die organischen Stoffe nutzten wir das Cauchy-Modell, das sehr gut für die durchsichtigen dünnen Filme passt. Die Realteile ( $n$ ) der komplexen Brechungsindizes der Dünnschichten variieren sich zwischen 2,2 und 3,4 , was höher als bei den meisten organischen und Polymerdünnfilmen ist. Die n-Werte vergrößern sich mit zunehmender Probendicke. Die Imaginärteile (k) der komplexen 
Brechungsindizes haben entgegengesetztes Verhalten. Man kann einen kleinen Peak in der Nähe von $330 \mathrm{~nm}$ aufgrund der Existenz von aromatischen Ringen sehen. Die Form der Spitzen ist schärfer geworden mit zunehmender Anzahl von Ringen.

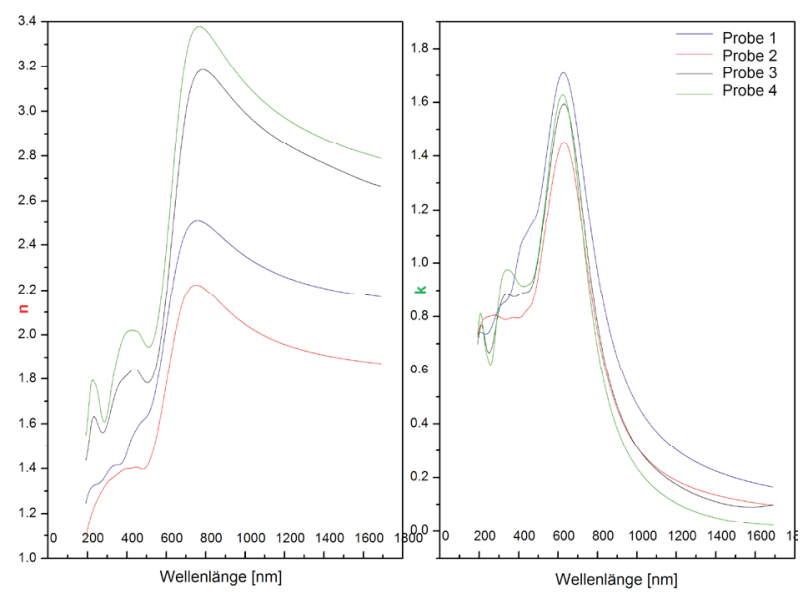

Abb.2: Optische Konstanten für die Proben 1-4.

Röntgen-Photoelektronenspektroskopie:

Diese Untersuchungen (XPS - Engl. X-Ray Photoelectron Spectroscopy) wurden mit einem ESCALAB250Xi von Thermo Scientific durchgeführt. Die Punktgröße der Röntgenquelle betrug $650 \mu \mathrm{m}$ und die Leistungswerte waren 14,8 $\mathrm{kV}$ und 13,7 mA. Die Durchgangsenergie des Analysators wurde auf $100 \mathrm{eV}$ für das UmfrageSpektrum und $20 \mathrm{eV}$ für die Elementspektren festgesetzt. Von allen Spektren wurde ein linearer Hintergrund abgezogen und die Bindungsenergie wurde auf $\mathrm{Au}=84,0 \mathrm{eV}$ kalibriert. Für die Filme wurden $\mathrm{Au}, \mathrm{C}$ und $\mathrm{S}$ gefunden.

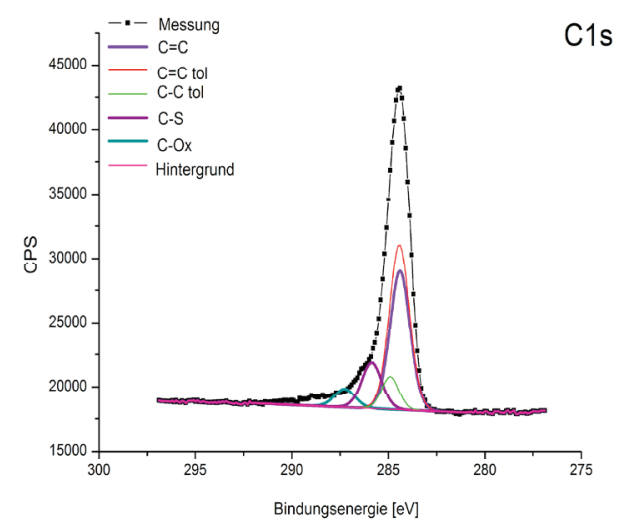

Abb. 3: C1s XPS Spektrum

Die Abwesenheit von Stickstoff zeigt, dass die stabilisierenden Moleküle des Dodecylamin von der Nanopartikelslösung mit den Linkermoleküle vollständig ausgetauscht sind. Die Spektren wurden unter Verwendung des Voigt-Profils $(30 \%$ Lorentz, Gauss 70\%) mit der Spin-Bahn-
Aufspaltung für Au4f und S2p gefittet. Das Intensitätsverhältnis war konstant - 4:3 (Au4f 7/2:Au4f 5/2) und 2:1 (S2p 3/2: S2p 1/2). Abb. 3 und Abb.4 zeigen die C1s und S2p XPS Spektren für die manuell hergestellten Proben.

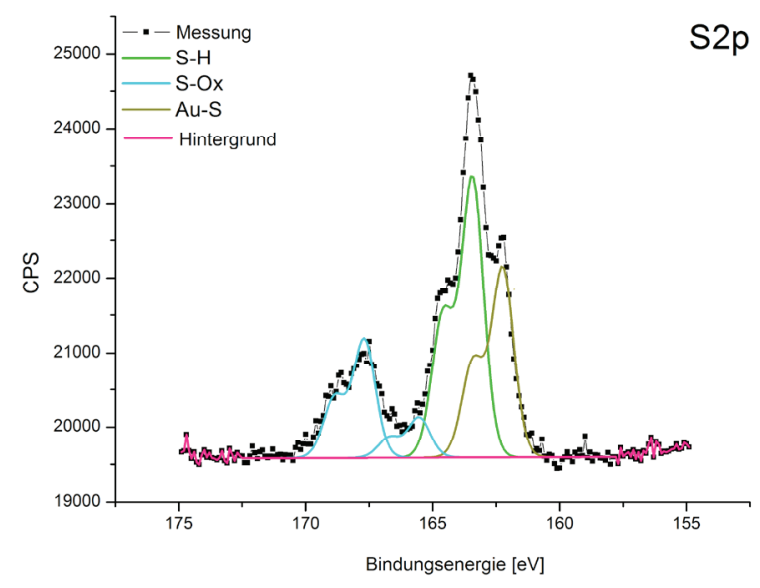

Abb. 4: S2p XPS Spektrum

Die gefundenen Elemente und entsprechende Peaks sind in Tab. 2 zusammengefasst.

Tab.2: Chemische Zusammensetzung.

\begin{tabular}{|l|l|}
\hline Bindungsenergie [eV] & \multicolumn{1}{|c|}{ Spezies } \\
\hline 162,$2 ; 163,4$ & S-Au \\
\hline 163,$4 ; 164,6$ & S-H \\
\hline 165,$5 ; 166,7$ & S-O $_{2}$ \\
\hline 167,$7 ; 168,9$ & S-Ox $(x>2)$ \\
\hline 284,$4 ; 284,43$ & C=C \\
\hline 284,9 & C-C \\
\hline 285,86 & C-S \\
\hline 287,23 & C-Ox \\
\hline
\end{tabular}

Das Vorhandensein von zusätzlichen zwei aromatischen und aliphatischen Peaks im C1s Spektrum sind von Restlösungsmittel verursacht, dass im Film verbleibt und sich zwischen die Nanopartikeln ähnlich wie die Dithiolmoleküle einlagert [10].

Von den Spektren in Abb. 3 und Abb. 4 kann man leicht sehen, dass die Anzahl der freien Thiole in den Filmen höher als die Anzahl der Schwefel-zu-Gold-Bindungen ist. Das zeigt, dass die Mehrheit des Dithioles entweder nur mit einem Ende an die Goldnanopartikel gebunden ist oder in der organischen Matrix eingelagert ist, statt kovalent an die Nanopartikeln gebunden zu sein. Solch eine Struktur der Nanokomposite kann durch kleine Längen von Linkermolekülen und eine breite Größenverteilung von Goldnanopartikeln zu Stande kommen. 
Oberflächenverstärkte Ramanspektroskopie:

Oberflächenverstärkte Ramanspektroskopie (SERS - Engl. Surface-enhanced Raman spectroscopy) wurde mittels eines RamanSpektrometers Labram HR 800 (Jobin Yvon) bei Raumtemperatur durchgeführt. Für alle RamanSpektren wurde die $532 \mathrm{~nm}(2,33 \mathrm{eV})$ Linie eines frequenzverdoppelten Nd:YAG-Laser mit der Leistung von 0,1 mW genutzt. Der Laserstrahl wurde auf die Probenoberfläche durch ein 50x Olympus Mikroskopobjektiv fokussiert. Das gestreute Licht wurde durch das gleiche Objektiv in Rückstreugeometrie gesammelt. Nach dem Durchlauf der Spektrometer mit Gittern von 600 Nutten/mm wurde das Streulicht mit einem thermoelektrisch gekühlten CCD-Detektor analysiert. Wir führten Messungen für die manuell vorbereitete Probe durch. Die Spektren des Filmes sind in Abb. 5. dargestellt.

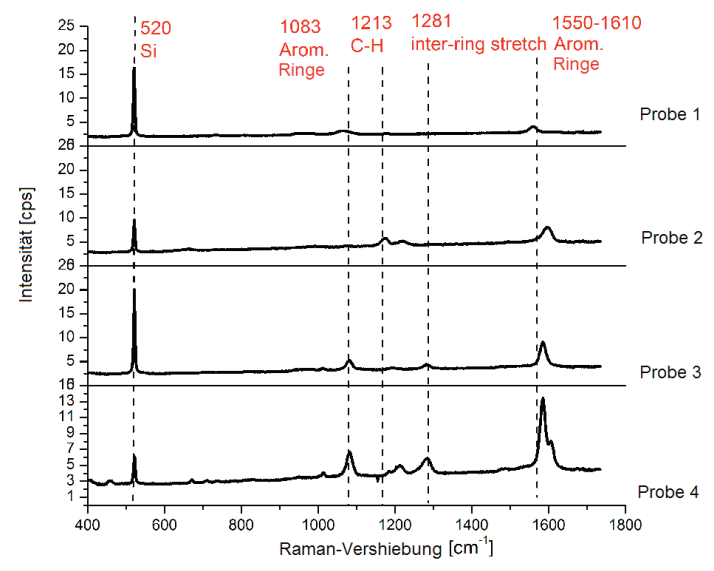

Abb. 5: SERS Spektren.

Im höheren Frequenzbereich können aromatische $\mathrm{C}-\mathrm{H}$-Bindungen detektiert werden, aber die Spitze ist sehr leicht abgeschrägt und unlesbar wegen der kleinen Menge von solchen Bindungen.

Die Intensität ist höher für die Filme, die mit Biphenyl-4,4'-dithiol und p-Terphenyl-4,4"-dithiol verlinkt sind. Das kann man im Hinblick auf die molekularen Polarisierung und Pre-Resonanz Theorie erklären $[10,11]$.

\section{Oberflächenverstärkte Infrarot-Absorption:}

Die SEIRA (Engl. - Surface-Enhanced Infrared Absorption) Spektren von Goldnanopartikelnfilme wurden mittels FTIR-TENSOR 27 (Bruker) Spektrometer in Transmissionregime unter Verwendung eines DLATGS-Detektors, mit einer Auflösung von $4 \mathrm{~cm}-1$ aufgezeichnet. Das Hintergrundspektrum des Siliziumsubstrats wurde gemessen und als Referenz verwendet. Die Spektren sind in Abb. 6 dargestellt.
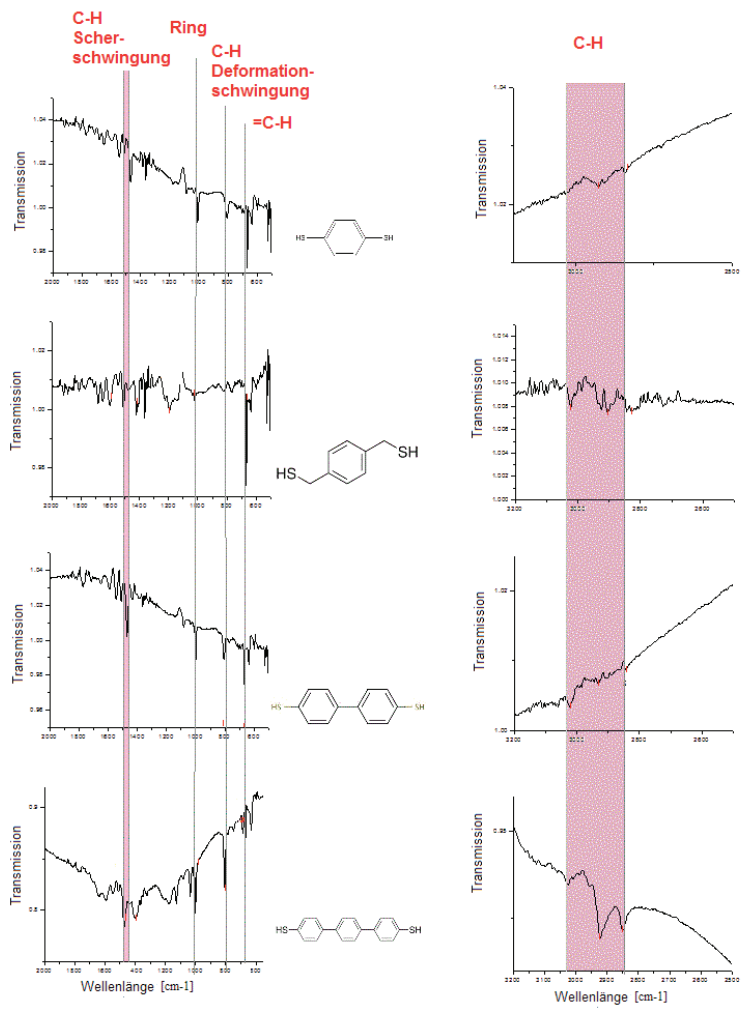

Abb. 6: SEIRA Spektren für die manuell hergestellten Filme.

Der Film verlinkt mit $p$-Terphenyl-4,4"-dithiol zeigt die höchste Signalverbesserung. Linkermoleküle innerhalb dieses Verbundes sind länger als in anderen Proben. Es ist bekannt aus der Literatur, dass die Verstärkung der Transmissionsspektren proportional zum Abstand zwischen den Metall-Nanopartikeln ist [12].

\section{Sensorische Messungen:}

Die Sorption des Toluols, 1-Propanols, 4-Methyl2-Pentanons und Wassers zu den organischen Molekülen-Nanopartikeln-Kompositen wurden durch Auftragen des Materials auf Substraten mit interdigitalen Elektroden untersucht. Die ähnlichen Dampfdrücke von Lösungsmitteln machen sie sehr gut geeignet für die Untersuchung der chemischen Selektivität von Sensormaterialien [1]. Die Sensorantworten für die Proben sind in Abb.7 zusammengefasst. Relativ hohe Empfindlichkeit kann durch die hohe Sorptionskapazität der löcherigen Film mit geringem Vernetzungsgrad erklärt werden. Eine Erhöhung des Widerstandes während der Experimente, d.h. positives elektrisches Signal ist mit dem Filmsschwellungsphänomen verbunden. Die Dampfempfindlichkeit von Au-basierten Chemoresistoren wurde im Hinblick auf die Schwellung und Porenausfüllung in den folgenden Publikationen diskutiert $[13,14]$. Es wurde darauf hingewiesen, dass die Diffusion des Analytes in die Poren die Dielektrizitätskonstante des Nanopartikels erhöht, während sehr geringe 
Schwellungen eine wesentliche Erhöhung des Filmwiderstands verursachen. Die Abnahme des Widerstands in den Proben 2-4 während der Experimente (d.h. negatives elektrisches Signal) kann durch das Phänomen der Porenausfüllung erklärt werden. Mit zunehmender Länge des Linkers erhöht sich die Porosität und das Porenausfüllungsphänomen. Gleichzeitig löst die Schwellung in diesem Fall nur eine geringe Wirkung aus. Die Selektivitäten sind für alle Materialien vergleichbar.

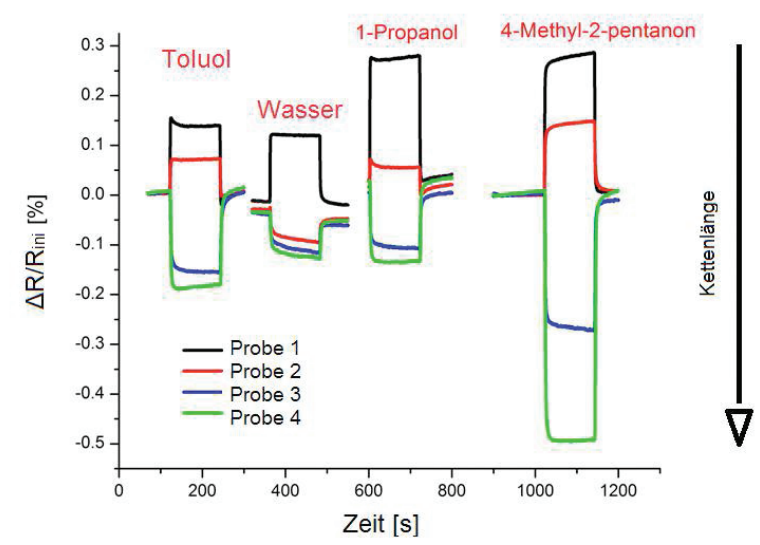

Abb. 7: Sensorantworten für mit einer Durchflußzelle vorbereitete Proben. Analytkonzentration - 5000 ppm.

Die stärkste Reaktion wurde für das flexible hydrophobe Molekül 4-Methyl-2-Pentanon beobachtet, schwächere Sensorantworten wurden für hydrophobes Toluol und polares 1-Propanol gemessen. Die schwächste Reaktion wurde für das polare Wassermolekül beobachtet. Abb.7 zeigt, dass alle Messungen recht schnell, aber nicht vollständig reversibel sind. Alle Reaktionen haben fast rechteckige Formen.

Abb. 8 fasst die Sensorantworten für die manuell-hergestellten Proben zusammen.

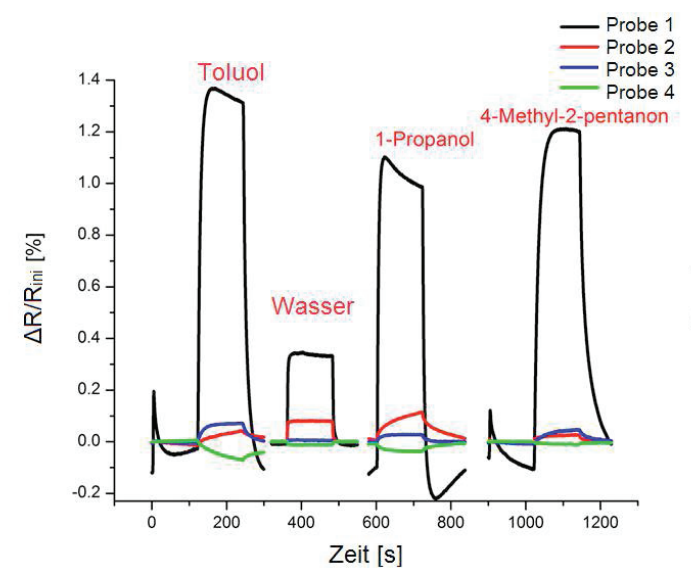

Abb. 8: Sensorantworten für die manuell vorbereitenen Proben. Analytkonzentration - 5000 ppm.
In diesem Fall sind die Antworten kleiner als bei den in der Durchflusszelle hergestellten Proben. Die Messungen waren langsamer und nicht vollständig reversibel. Nur die Probe mit pTerphenyl-4,4"-dithiol verlinkt zeigt eine negative Antwort auf 1-Propanol und Toluol. Das bedeutet, dass für die manuell hergestellten Proben der Hauptsensormechanismus Schwellung ist. Andererseits steigt die Porenmenge noch mit zunehmender Länge des Linkers an.

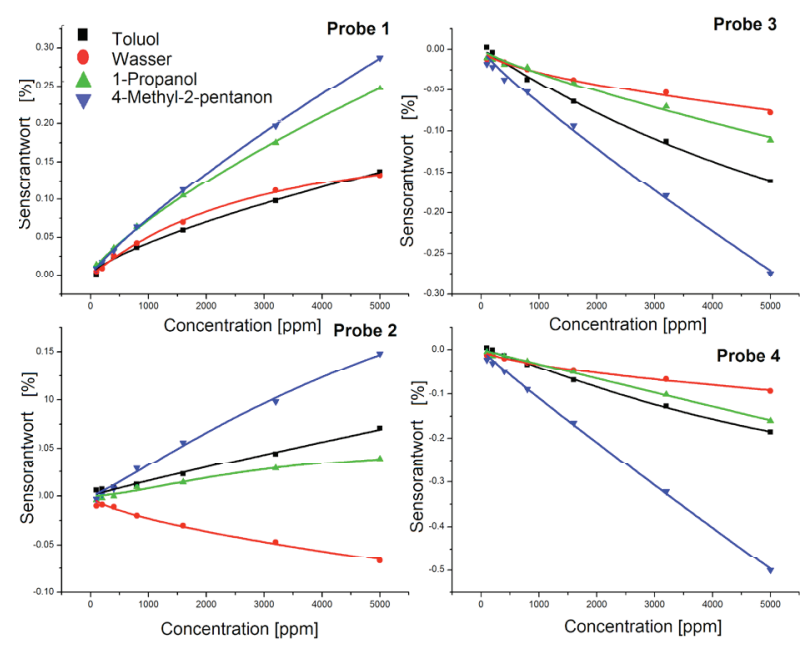

Abb. 9: Langmuir Fits der resistiven Antwortisothermen. Die Proben wurden mit der Durchflußzelle hergestellt.

Für alle Analyte wurden die konzentrationsabhängigen Massemessungen im Bereich von 100 - 5000 ppm durchgeführt. Die Isothermen sind in Abb. 9 und Abb.10 dargestellt. Die Sorptionisothermen können perfekt mit Langmuir Formalismus gefittet werden. Das zeigt, dass die Wechselwirkungen der verschiedenen Analyten mit dem Film ähnlich sind.
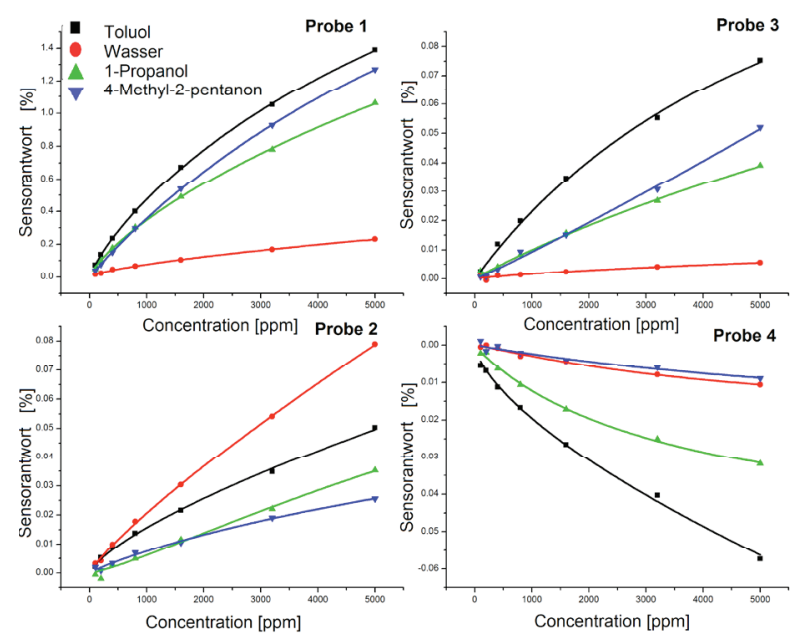

Abb. 10: Langmuir Fits der resistiven Antwortisothermen. Proben wurden manuell hergestellt. 


\section{Literatur}

[1] JOSEPH, Y. ; BESNARD, I. ; ROSENBERGER, M. ; GUSE, B. ; NOTHOFER, H-G.; WESSELS, J. M. ; WILD, U. ; KNOP-GERICK, A. ; SU, D. ; SCHLÖGL, R. ; YASUDA, A. ; VOSSMEYER, T.: Self-Assembled Gold Nanoparticle/Alkanedithiol Films: Preparation, Electron Microscopy, XPS-Analysis, Charge Transport, and Vapor-Sensing Properties. In: J. Phys. Chem. B 107 (2003), Nr. 107, S. 7406-7413

[2] JOSEPH, Y. ; GUSE, B. ; VOSSMEYER, T. ; YASUDA, A.: Gold Nanoparticle/Organic Networks as Chemiresistor Coatings: The Effect of Film Morphology on Vapor Sensitivity. In: J. Phys. Chem. C 112 (2008), Nr. 21, S. 12507-12514

[3] TODA, M. ; JOSEPH, Y. ; BERGER, R.: Swelling of Composite Films at Interfaces. In: J. Phys. Chem. C 114 (2010), Nr. 5, S. 2012-2017

[4] PENG, S. ; LEE, Y. ; WANG, C. ; YIN, H. ; DAI, S. ; SUN, S.: A Facile Synthesis of Monodisperse Au Nanoparticles and Their Catalysis of CO Oxidation. In: Nano research 1 (2008), Nr. 3, S. 229-234

[5] SCHLICKE, H. ; SCHRÖDER, J. H. ; TREBBIN, M. ; PETROV, A. ; IJEH, M. ; WELLER, H. ; VOSSMEYER, T.: Freestanding Films Of Crosslinked Gold Nanoparticles Prepared Via Layer-by-Layer SpinCoating. In: Nanotechnology 22 (2011), Nr. 30, S. 305303-305312

[6] DASKAL, Y. ; DITTRICH, R. ; HIMCINSCHI, C. ; ABENDROTH, B. ; WALTER, J. ; JOSEPH, Y.: Novel Gold Nanoparticle Organic Composites: Characterization of Optical and Sensing Properties, In: Proceedings AMA Conferences 2015, AMA Conferences 2015 Sensor 2015 And IRS ${ }^{2}$. Nuremberg, Germany, 19-21 May 2015

[7] BETHELL, D. ; BRUST, M. ; SCHIFFRIN, D. J. ; KIELY, C.: From Monolayers to Nanostructured Materials: an Organic Chemist's View of Self-Assembly. In: J. Electroanal. Chem. 409 (1996), Nr. 1-2, S. 137-143

[8] KONDO, M. ; TADA, T. ; YOSHIZAWA, K.: J. Phys. Chem. A 108 (2004), Nr. 42, S. 9143-9149

[9] KALA, C. P.: Study of Electron Transport Phenomena through Molecules for Nano - Scale Electronic Devices, SRM Institute of Science and Technology, Doctoral thesis, 2013

[10] SANTOS, P. S. ; FARIA, D. L. A. ; SANTOS, J. G. N.: The Pre-resonance Raman Effect of the Species $\mathrm{I}^{-} \cdot \mathrm{SO}_{2}$ and $\mathrm{SCN}^{-} \cdot \mathrm{SO}_{2}$. In: J. Mol. Struct. 161 (1987), S. $15-22$

[11] OHTA, N. ; ITO, M.: Pre-resonance Raman effect and Vibronic Coupling of Benzene and Benzened6. In: Chem. Phys. 24 (1977), Nr. 2, S. 175-181

[12] VERGER, F. ; PAIN, T. ; NAZABAL, V. ; BOUSSARD-PLÉDEL, C. ; BUREAU, B. ; COLAS, F. ; RINNERT, E. ; BOUKERMA, K. ; COMPĖRE, S. ; GUILLOUX-VIRY, M. ; DEPUTIER, S. ; PERRIN, A. ; GUIN, J. P.: Surface Enhanced Infrared Absorption (SEIRA) Spectroscopy Using Gold Nanoparticles on $\mathrm{As}_{2} \mathrm{~S}_{3}$ Glass. In: Sens. \& Acts. B 175 (2012), S. 142-148

[13] VOSSMEYER, T. ; JOSEPH, Y. ; BESNARD, I. ; HARNACK, O. ; KRASTEVA, N. ; GUSE, B. ;
NOTHOFER, H.-G. ; YASUDA, A.: GoldNanoparticle/Dithiol Films as Chemical Sensors and First Steps toward their Integration on Chip. In: Proc. SPIE, Physical Chemistry Of Interfaces And Nanomaterials III, Denver, Colorado, USA, 3-6 August 2004

[14] VOSSMEYER, T. ; GUSE, B. ; BESNARD, I. ; BAUER, R. E. ; MÜLLEN, K. ; Yasuda, A.: Gold Nanoparticle/Polyphenylene Dendrimer Composite Films: Preparation and Vapor-Sensing Properties. In: Adv. Mater. 14 (2002), Nr. 3, S. 238-242 